\title{
A NEW STRIPLINE MEASURING SETUP FOR THE CHARACTERISATION OF CONDUCTIVE GASKETS UP TO $18 \mathrm{GHz}$
}

\author{
Johan Catrysse*, Filip Vanhee, Davy Pissoort, Christian Brull** \\ KHBO, Flanders Mechatronics Engineering Center \\ Zeedijk 101, B 8400 Oostende (Belgium) \\ *MICAS/ESAT, KULeuven \\ Kasteelpark Arenberg 10, B 3001 Heverlee-Leuven (Belgium) \\ ** SEM Schlegel Electronic Materials, Slijpesteenweg 28, B 8432 Leffinge (Belgium)
}

\begin{abstract}
Due to the impact of higher and higher frequencies, the Shielding Effectiveness (SE) characterisation of shielding gaskets at frequencies above $1 \mathrm{GHz}$ is needed for on board shielding applications. Although the standard IEEE Std $1302^{\mathrm{TM}}-2008$ is covering the characterisation of shielding gaskets up to $18 \mathrm{GHz}$, the methods proposed in this standard are not applicable for these thin and small 'on board' gaskets. A method overcoming this problem is proposed in this paper.
\end{abstract}

\section{Keywords: conductive gaskets, shielding}

\section{INTRODUCTION}

Due to the impact of higher and higher frequencies, the SE characterisation of shielding gaskets at frequencies above $1 \mathrm{GHz}$ is needed for on board shielding applications. On board shielding is an application where noisy components must be shielded, in order to cause no interference with the environment (far field) or with adjacent electronic components (near field). The quality of shielding strongly depends on the way conductive contact is made from the small enclosure (CAN) to the ground plane of the PCB. In some cases, a thin gasket is needed to ensure good conductive contact. Although the standard IEEE Std $1302^{\mathrm{TM}}-2008$ is covering the characterisation of shielding gaskets up to 18 $\mathrm{GHz}$, the methods proposed in this standard are not applicable for these type of thin and small gaskets, and the problem is only shortly addressed in the Annex E of this standard [1] and shown in figure 1.

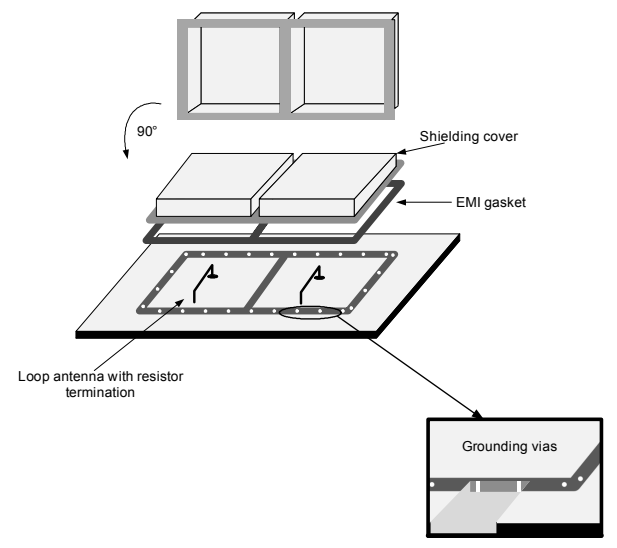

Figure 1. Typical configuration of on board shielding

\section{MEASURING METHODS}

Actually, a number of possible measuring methods are proposed and discussed in literature [2] - [6]. Most of them are based on a modified MIL-DTL$83528 \mathrm{C}-2001$ methodology [7], or the use of a reverberation chamber, or near field measurements.

The general setup for MIL-DTL-83528C-2001 methodology [7] and similar configurations is shown in figure 2. A hole is made in one wall of a shielded (anechoic) room, and a plate is clamped against this opening, compressing a gasket in between the clamping plate and the wall.
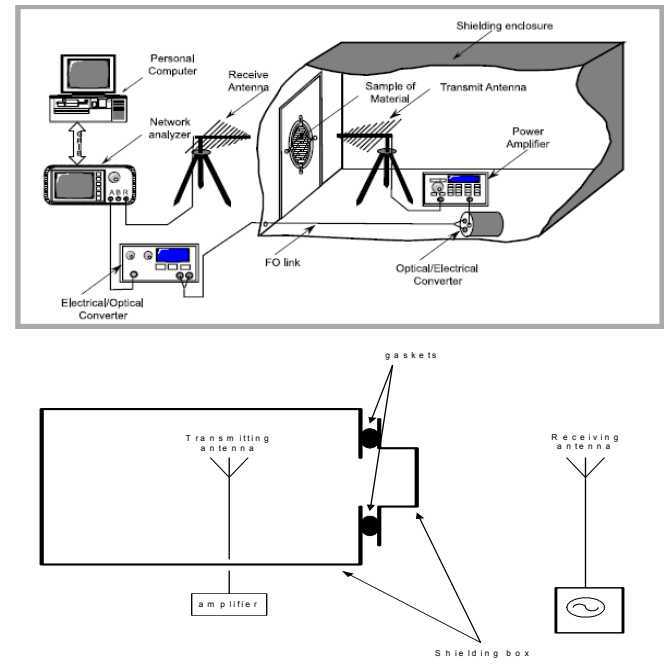

Figure 2. Global and detailed view of MIL DTL 83528C setup and similar configurations

Although a well established measuring method, it is not well suited for the characterization of small gaskets intended for on board shielding use. The setup requires a large opening (at least $50 \times 50 \mathrm{~cm}$ ) to be covered, and consequently large samples, which are normally not available for these gaskets.

Another method is based on measurements performed in a reverberation chamber [6]. Different approaches can be used for setting up a measuring configuration in a reverberation chamber. One is using a horn antenna at higher frequencies, another one is using a small monopole probe, mounted on one of the walls of the reverberation chamber. The latter one is shown in Fig. 3. 


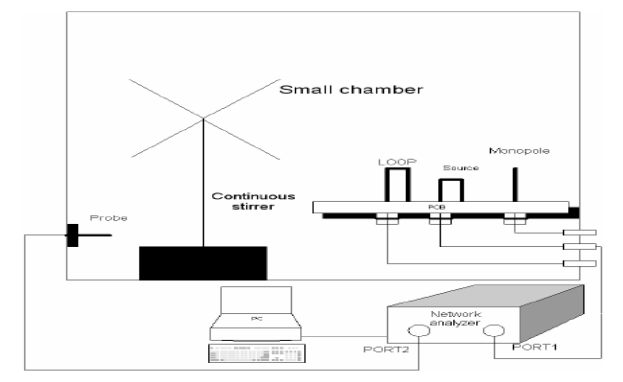

Figure 3: Configuration of a mode stirred reverberation chamber, using a monopole in the frequency range $1-6 \mathrm{GHz}$.

In this case, small loop and/or monopole antenna's are used as transmitting sources or receivers. The main problem is the fact that these small antenna's are not matched to $50 \mathrm{Ohm}$ in the frequency range of interest.

Instead of the environment of a stirred reverberation chamber and its antenna configuration, a second set of on-board antenna's can be used. The equivalence of measured SE values between reverberation chamber setup and near field testing, has been reported in [6].

Due to the small size of the antenna's, the method has a restricted dynamic range, which might imply the use of a high power amplifier.

Furthermore, these methods does not fit directly to an on-board application of the gasket for PCB level shielding.

\section{NOVEL MEASURING METHOD}

In order to overcome some of the disadvantages mentioned in section 2, another setup has been developed.

Recently, an interesting test methodology has been proposed for susceptibility and emission testing of IC's: IC stripline method [8]-[10]. The method is based on putting a stripline over a PCB board with a full GND layer, so that susceptibility or emission of an Integrated Circuit may be performed. The principle of the setup is sketched in figure 4 .

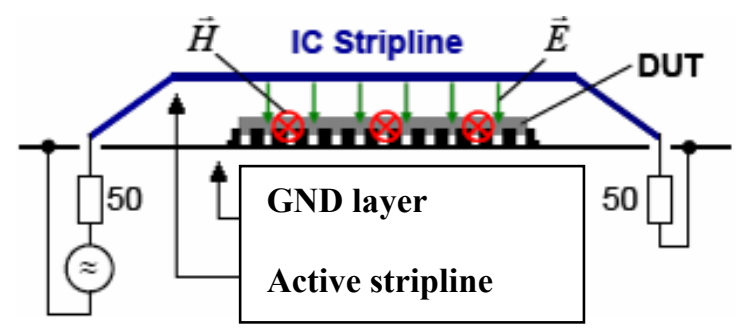

Figure 4. Principle setup of the proposed stripline method in order to characterize IC's

By replacing the IC under test by a $50 \mathrm{Ohm}$ microstrip, and a plate to clamp and compress a gasket to the chassis of the system, a matched measuring setup is obtained, with a direct relationship to the physical and mechanical environment of on-board shielding.
The proposed measuring setup is shown in the next figures $5-8$ and has an overall size of $17 \times 20 \mathrm{~cm}$.

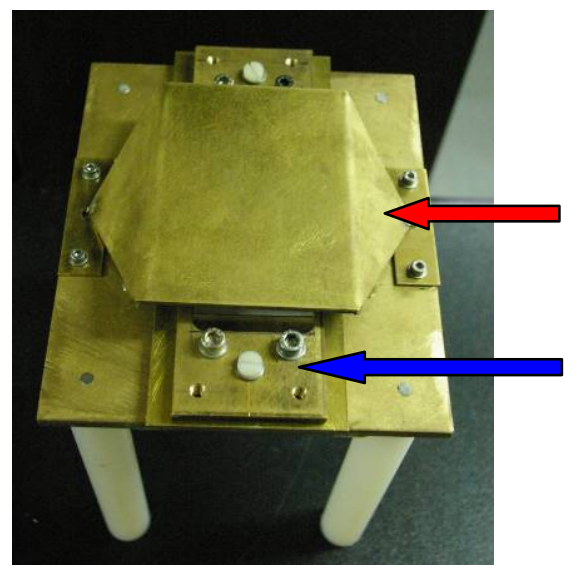

Figure 5. Overall view of the stripline setup

A stripline has been designed to fit a characteristic impedance of $50 \mathrm{Ohm}$ (red arrow). The width of the stripline is $12 \mathrm{~cm}$ and the height above the solid GND copper plate is $2.6 \mathrm{~cm}$. The active length of the stripline is $9 \mathrm{~cm}$, and both tapering sections are $3 \mathrm{~cm}$ each. The inner side of the stripline is covered with an absorbing ferrite sheet, to avoid too much influence of the short tapering. SMA connectors are mounted through the solid GND plate.

Another solid copper plate is intended to hold the gasket, and is inserted in the open area of the stripline (blue arrow).

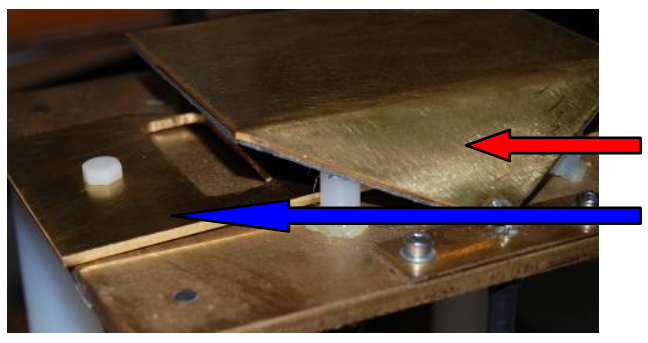

Figure 6. Detailed view of the stripline setup

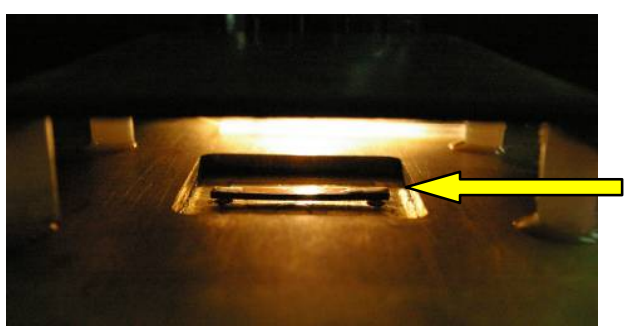

Figure 7. Embedded microstrip

Embedded in the solid GND plate, there is a small $50 \mathrm{Ohm}$ microstrip, with a matched load of $50 \mathrm{Ohm}$ connected via an SMA connector through the GND plate. This microstrip is used as the transmitting antenna, and simulates the radiating traces on a virtual PCB. The microstrip has a length of $4 \mathrm{~cm}$ and is made on a substrate of microwave PCB material, and is embedded in a $5 \times 5 \mathrm{~cm}$ opening. 
By covering this embedded microstrip with a plate or sheet, the Shielding Effectiveness (SE) of this material can be evaluated, by performing two measurements: a first coupling between microstrip and stripline in an open structure and a second one when covered with the material.

Using a solid copper plate to cover the embedded microstrip, and inserting a gasket in between this plate and the solid GND plate of the stripline structure, the SE of the gasket can be measured. In this way, the copper plate can act as a gasket sample holder, and the gasket may be carefully positioned on this solid plate. This is shown in figure 8 . When placing this sample holder in place, the embedded microstrip is exactly within the inner surface area limited by the gasket.

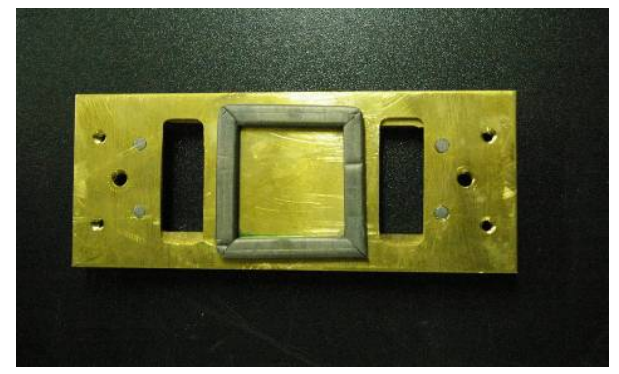

Figure 8. Solid copper plate acting as gasket sample holder

Both the microstrip and the stripline have one matched load of $50 \mathrm{Ohm}$, so that the setup may be considered as a 2-port circuit.

\section{PRELIMINARY VALIDATION (8 GHz)}

Due to the availability of measuring equipment, a preliminary validation of the system was performed up to the frequency of $8 \mathrm{GHz}$. For these measurement purposes, a vector network analyser (VNA) has been used, which enables the measurement of the 4 S-parameters of the system. The $4 \mathrm{~S}$-parameters for the open system are given in figure 9, showing the relatively fair $50 \mathrm{Ohm}$ design (S11 and S22) and the coupling between the microstrip and the stripline (S21).

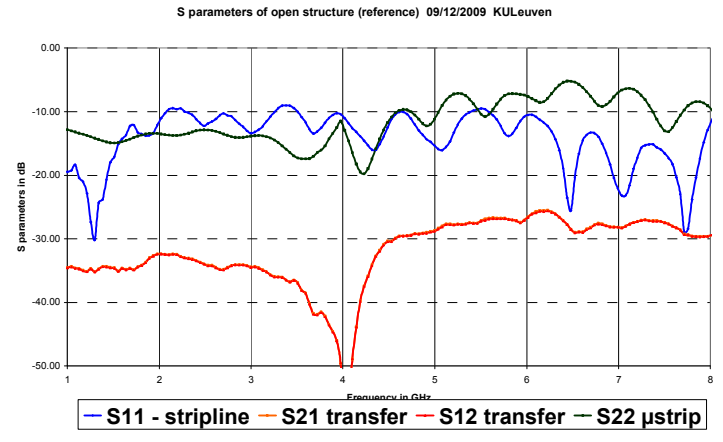

Figure 9. S-parameters of the stripline setup

A sharp resonance is observed around $4 \mathrm{GHz}$, and which can not be directly attributed to a typical dimension of the system.
For the purpose of this paper, an example of the obtained measuring results is reported for one type of gasket: Dynashear ${ }^{\mathrm{TM}}$ from SEM.

The VNA allows to calibrate the measured S21 parameter of the open structure as reference, so that further measurements of S21 will directly show the SE levels of the configuration under test. The gasket was fixed on the sample holder, as can be seen on the picture of figure 8 .

Three different cases have been tested:

- inserting the sample holder, without further compression of the gasket (green line)

- compressing the gasket down to a thickness of $1 \mathrm{~mm}$ (blue line)

- $\quad$ inserting an insulating paper sheet between the compressed gasket and the solid base plate, so that no conductive contact is realised (red line)

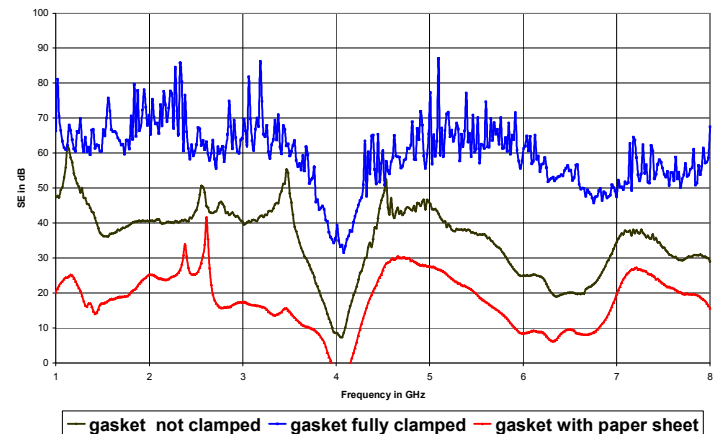

Figure 10. Typical set of SE values

Three main effects may be observed:

- a clear discrimination between the 3 different cases, showing the importance of making and maintaining a good conductive contact between both parts of a shielding system

- even by calibrating out the sharp resonant effect in the open structure, it is still present when performing the SE measurements

- when a high level of SE is obtained (fully compressed gasket), the dynamic range of the measuring equipment is exceeded, and a very noisy result is obtained. This might be overcome by the use of an extra amplifier in the transmitting chain. The effect of using an amplifier providing an extra $20 \mathrm{~dB}$ gain, and the mechanical adaptations as discussed and shown in figure 12, is given in figure 11.

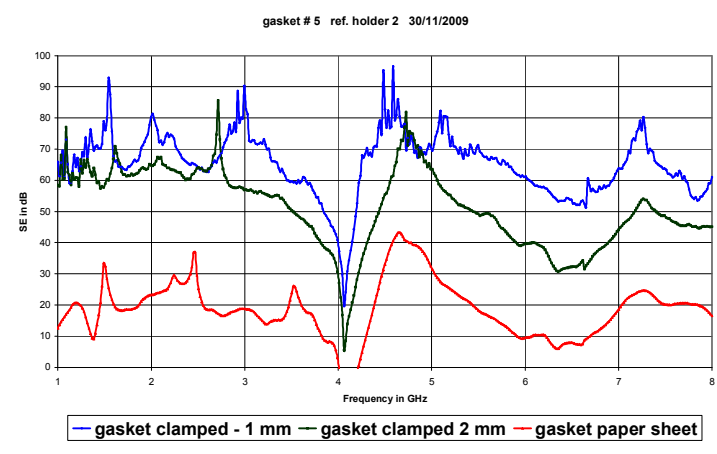

Figure 11. Measured SE values, with an extra $20 \mathrm{~dB}$ amplifier 
Other mechanical problems were identified and corrected in a final version of the setup. First of all, in order to ensure that no conductive contact could occur when the sample holder was screwed to the base plate, plastic screws were used. However, due to the high forces needed to fully compress the gaskets, they show some elongation because of the elasticity of the plastic.

To ensure no conductive contact at the screwing area, insulating sheets were mounted, and metal bolts are now used, within a small plastic tube. The exact compression is now controlled using plastic spacers of different thickness, that can be inserted between the sample holder and the base plate.

The new mechanical design is shown in the next pictures.
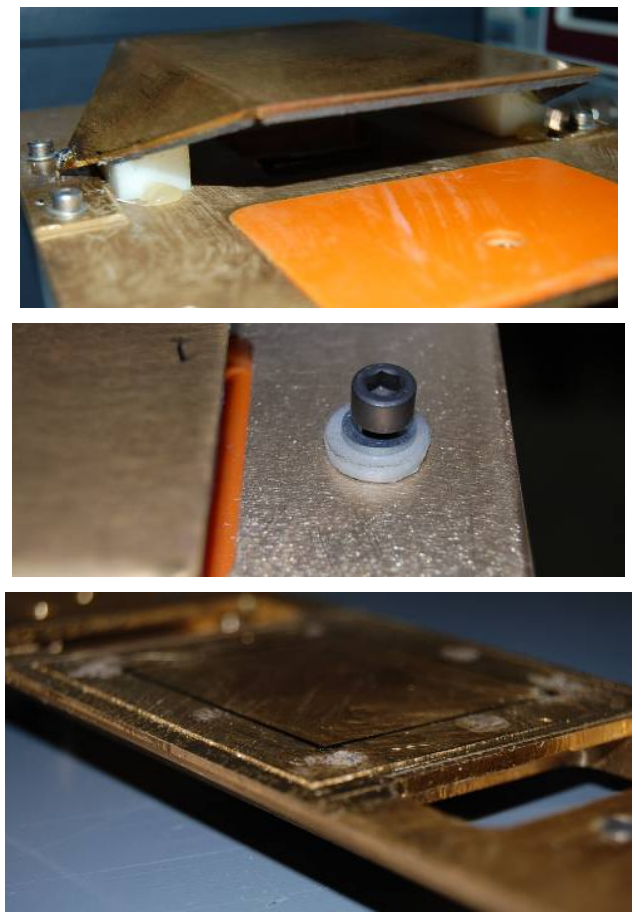

Figure 12. View of the insulating sheet, to avoid conductive contact between holder and base plate (upper), metal bolt in a plastic tube to compress the gasket (middle) and visible alignment for exact positioning of the gasket (lower)

A first validation of the upgraded version of the stripline fixture, including the use of an amplifier, was already reported in figure 11 .

The sharp resonance around $4 \mathrm{GHz}$ is still observed. First of all, as the stripline is a kind of open TEM cell structure, this effect might be due to the generation of higher order modes and/or the occurance of relatively high radiation „loss”. Also, effects of reflections by nearby structures, and coupling back in the system due to its open structure, should not be excluded. Appropriate experimental work showed no relevant influence due to the open structure. The possible effect of higher order modes should dissapear, when taking the reference S21 measurement to calibrate the setup. Which is clearly not the case, as can be seen from figures 10 and 11 .
As the length of the microstrip is $4 \mathrm{~cm}$, and even the "effective" length is only $3.6 \mathrm{~cm}$ (distance between the soldering points of the SMA connectors), it looks like an effect due to some resonant pattern of the microstrip itself at half a wavelength.

Some optimisation of the microstrip structure has been done, so that the whole ground plane is now covered with the PCB dielectric material (red arrow) and the structure tends better to a homogenous $\mu$ strip. And some absorbing material has been mounted on the "walls" of the embedded structure (blue arrow). This is shown in figure 13.

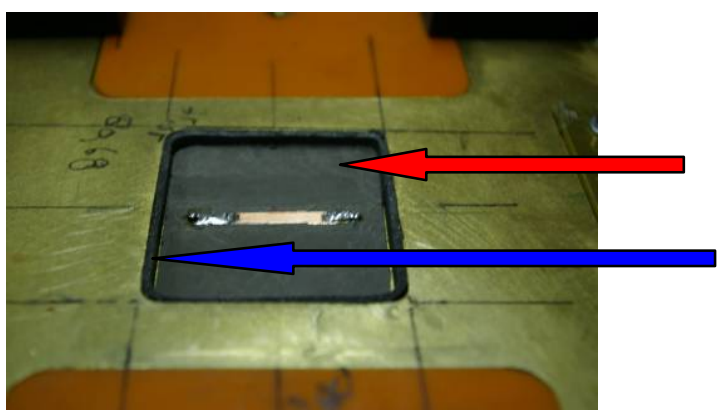

Figure 13. Optimising the embedded microstrip structure to avoid resonant effects.

The open structure can be modelled as a kind of directional coupler made of asymmetrical coupled lines, as sketched in figure 14

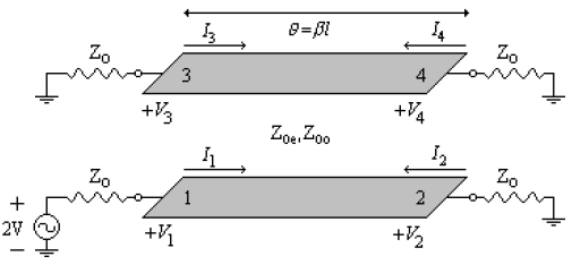

Figure 14. Schematic figure of a directional coupler

It is a well known characteristic of these geometries that sharp uncoupling at one of the ports may occur at a given frequency. Also, a different behaviour of the coupled ports \#3 and \#4 is known as the Near End and the Far End coupling respectively [11].

Both simulation and measurement show the difference of the coupling to both ports. Simulation has been done with air as dielectric material, as no good parameters were available for the PCB.

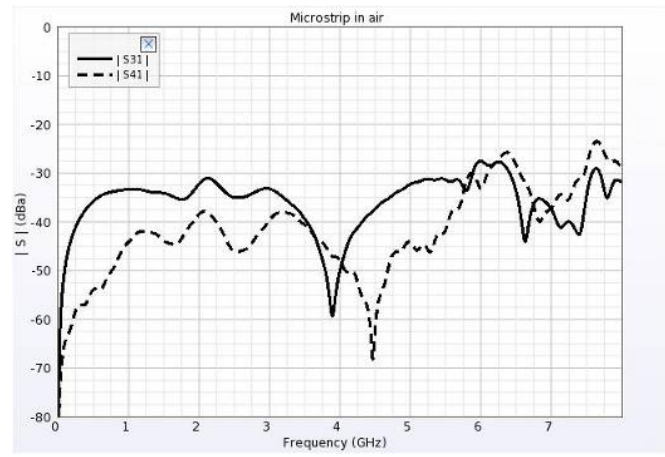

Figure 15. Simulation of directional coupler: S31 \& S41 
The results are given in figures 15 (simulation) and figure 16 (measurement).

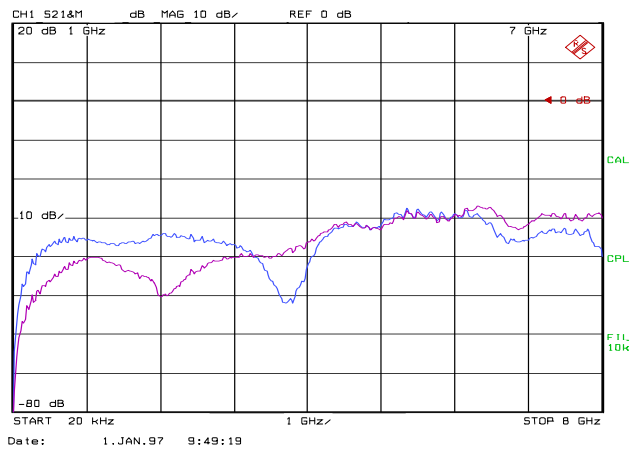

Figure 16. Measured S31 \& S41 parameters

Due to the dielectric losses and the absorbing material, some resonant effects are smoothed out for the measured parameters.

In order to measure the shielding performance of a gasket, the VNA is now calibrated using the parameter of the open structure as a reference.

The shielding performance of the reference gasket has been measured, under three conditions:

- $\quad$ clamping distance of $2 \mathrm{~mm}$ (gasket making nearly no contact with the metal reference GND plate) - blue line in figure 17

- $\quad$ clamping distance of $1 \mathrm{~mm}$ (gasket nearly fully compressed) - red line in figure 17

- paper sheet inserted, with a compression of the gasket at $1 \mathrm{~mm}$ (no conductive contact) - green line in figure 17

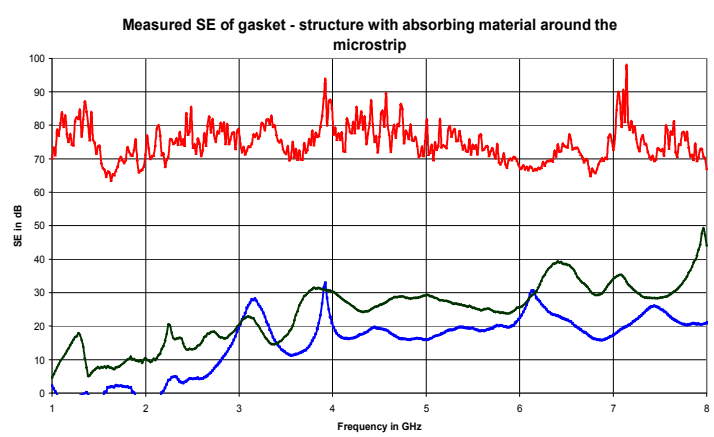

- gasket $5 \cdot 2 \mathrm{~mm}$ (not) clamped $\rightarrow$ gasket $5.1 \mathrm{~mm}$ clamped $\rightarrow$ gasket $5.1 \mathrm{~mm}$ clamped paper sheet

Figure 17. SE of the gasket under different conditions of compression and conductive contact

First of all, it is observed that the sharp resonant effects have disappeared, which gives now a smooth curve for the measured SE values.

The effect of loss of conductive contact is clearly observed, in case of an insulated surface or lack of compression. It is also observed that even using a $20 \mathrm{~dB}$ amplifier in the transmitting path, the dynamic range is still reached in case of a high performing shielding gasket. This is seen from the noisy aspect of the measured SE curve.

\section{FINAL VALIDATION UP TO $18 \mathrm{GHz}$}

A final validation of the stripline fixture has been performed with an appropriate VNA up to $18 \mathrm{GHz}$. It means that the dynamic range of the measuring setup is limited, and that high performing shielding gaskets will show a noisy measuring result. Unfortunately, at the moment of these measurements, no amplifier up to this frequency was available.

The S-parameters of the stripline fixture are given in figure 18 and a first set of SE results in figure 19.

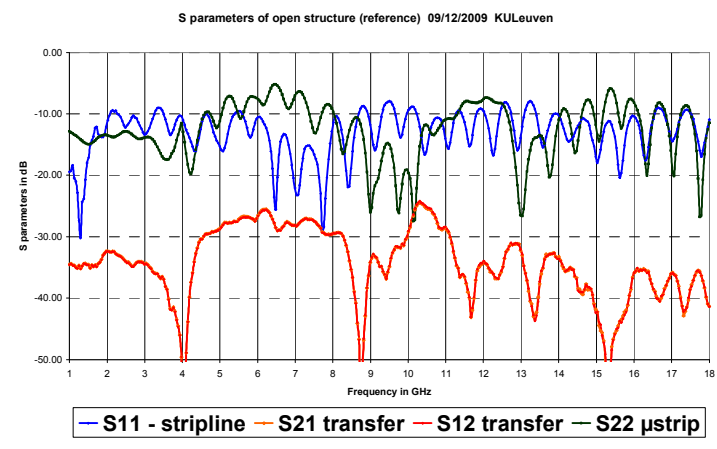

Figure 18. S-parameters of the stripline setup

As an example, the measured SE values of the same type of gasket as used for the preliminary testing, are given in figure 19.

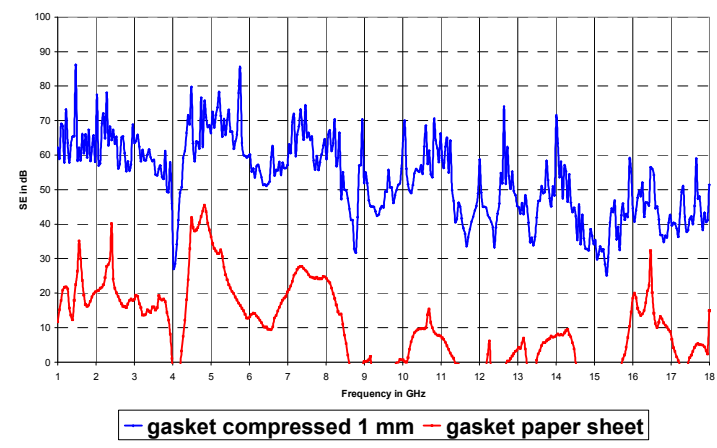

Figure 19. First validation of the setup, up to $18 \mathrm{GHz}$

\section{CONCLUSIONS}

A novel compact and easy to handle test fixture for the characterization of on-board PCB shielding gaskets up to a frequency of $18 \mathrm{GHz}$, has been discussed and validated.

Future work is still needed on the modelling of the setup as a directional coupler using asymmetrical, weakly coupled transmission lines.

More future work is needed about the repeatability of the method and the critical parameters in the handling of the system. Final validation of the methodology must be done by simulation and comparison to other measuring methods. 
The proposed measuring setup shows clearly the effects of non-contacting situations when applying conductive gaskets, and offers a very valuable engineering tool, in order to get SE values that can directly applied when designing on-board shielding enclosures at PCB level.

\section{References}

[1] IEEE Std 1302 Electromagnetic Characterization of Conductive Gaskets in the Frequency Range of DC to $18 \mathrm{GHz}$ ”, IEEE, nov. 2008

[2] Sarto, M. S., et al., "An Innovative Shielding Concept for EMI Reduction,” IEEE EMC Society Newsletter, Summer 2001.

[3] Clupper T., "Effects of gaps in multiple EMI enclosures on RF PCBs," Proceedings of the IEEE International Symposium on EMC, Montreal, Canada, Aug. 2001.

[4] "Near-Field Shielding Effectiveness and Cavity Isolation Test Plan,” TP3375.02, Chomerics, Woburn, MA, 15 Jan. 2002.
[5] F. Vanhee, J. Catrysse et al., "Proposed methods to measure the shielding performance of PCB level enclosures", Proc. Int. Symp.on EMC, Kyoto, July 2009

[6] He Yuhui and A. Marvin, "An investigation of the shielding performance of PCB level enclosures using reverberation chamber", Proc. IEEE Int. Symp. on EMC, Hawai, Aug. 2007

[7] MIL-DTL-83528C-2001, "General Specification

for Gasketing Material, Conductive, Shielding Gasket, Electronic, Elastomer, EMI/RFI", 2001

[8] B. Koerber et al., "IC-stripline: a new proposal for susceptibility and emission testing of IC's", Proceedings EMCcompo 2007

[9] ISO 11452-5, Road Vehicles, Component test methods for electrical disturbances from narrowband radiated EM energy part 5: stripline method.

[10] Wadell, B., “Transmission line design handbook", Artech House 1991

[11] Cristal E. "Coupled Transmission Line Directional Couplers with Coupled Lines of Unequal Characteristic Impedances", IEEE Trans. on MTT, vol. 14, nr. 7, pp. 337-346, July 1966. 